\title{
Formal Verification of Safety \& Security Related Timing Constraints for a Cooperative Automotive System
}

\author{
Li Huang ${ }^{1}$ and Eun-Young Kang ${ }^{2(\bowtie)}$ \\ 1 School of Data and Computer Science, Sun Yat-Sen University, Guangzhou, China \\ huang1223@mail2. sysu.edu.cn \\ 2 The Maersk Mc-Kinney Moller Institute, University of Southern Denmark, \\ Odense, Denmark \\ eyk@mmi.sdu.dk
}

\begin{abstract}
Modeling and analysis of timing constraints is crucial in realtime automotive systems. Modern vehicles are interconnected through wireless networks which creates vulnerabilities to external malicious attacks. Violations of cyber-security can cause safety related accidents and serious damages. To identify the potential impacts of security related threats on safety properties of interconnected automotive systems, this paper presents analysis techniques that support verification and validation $(\mathrm{V} \& \mathrm{~V})$ of safety \& security $(\mathrm{S} / \mathrm{S})$ related timing constraints on those systems: Probabilistic extension of S/S timing constraints are specified in PrCCSL (probabilistic extension of clock constraint specification language) and the semantics of the extended constraints are translated into verifiable UPPAAL models with stochastic semantics for formal verification. A set of mapping rules are proposed to facilitate the translation. An automatic translation tool, namely ProTL, is implemented based on the mapping rules. Formal verification are performed on the S/S timing constraints using UPPAAL-SMC under different attack scenarios. Our approach is demonstrated on a cooperative automotive system case study.
\end{abstract}

Keywords: Automotive system - Safety and security $\cdot$ PrCCSL $~$ UPPAAL-SMC

\section{Introduction}

Model based development (MBD) is rigorously applied in automotive systems in which the software controllers interact with physical environments. The continuous time behaviors of those systems often rely on complex dynamics as well as on stochastic behaviors. Formal verification and validation (V\&V) technologies are indispensable and highly recommended for development of safe and reliable automotive systems $[11,12]$. Conventional V\&V, i.e., testing and model checking have limitations in terms of assessing the reliability of hybrid systems due to both stochastic and non-linear dynamical features. To ensure the reliability of safety

(C) The Author(s) 2019

R. Hähnle and W. van der Aalst (Eds.): FASE 2019, LNCS 11424, pp. 210-227, 2019.

https://doi.org/10.1007/978-3-030-16722-6_12 
critical hybrid dynamic systems, statistical model checking (SMC) techniques have been proposed $[7,8,19]$. These techniques for fully stochastic models validate probabilistic performance properties of given deterministic (or stochastic) controllers in given stochastic environments.

Modern vehicles are being equipped with communication devices and interconnected with each other through wireless networks. Vehicular Ad Hoc Networks (VANET) [28] are the technologies of wireless networks that establish communication among vehicles and roadside units (RSU). Nevertheless vehicular communication contributes to the safety and efficiency of traffic, it introduces vulnerabilities to vehicles. Transmitted information can be corrupted or modified by attackers, resulting in serious safety consequences (e.g., rear-end collision). Analysis of the potential impacts of cyber-security violations on safety properties is crucial in automotive systems. However, traditional automotive system design often addresses the correctness of safety properties without consideration of security breaches. There is still a lack of techniques that enable an integrated analysis of safety \& security $(\mathrm{S} / \mathrm{S})$ properties. Moreover, message transmission in VANET that pertains to $\mathrm{S} / \mathrm{S}$ requires restrictions by time deadlines [10]. In this paper, we focus on $\mathrm{S} / \mathrm{S}$ related timing constraints and propose analysis techniques that support formal verification on interconnected automotive systems.

EAST-ADL $[9,22]$ is an architectural description language for modeling of automotive systems. The latest release of EAST-ADL has adopted the time model proposed in Timing Augmented Description Language (TADL2) [5], which expresses and composes basic timing constraints, i.e., repetition rates, end-to-end delays. TADL2 specializes the time model of MARTE, the UML profile for Modeling and Analysis of Real-Time and Embedded systems [30]. MARTE provides CCSL, a Clock Constraint Specification Language, that supports specification of both logical and dense timing constraints, as well as functional causality constraints [16,23]. A probabilistic extension of CCSL, called PrCCSL [14], has been proposed to formally specify timing constraints associated with stochastic properties in weakly-hard real-time systems [4], i.e., a bounded number of constraints violations would not lead to system failures when the results of the violations are negligible.

In this paper, we present a formal analysis of $\mathrm{S} / \mathrm{S}$ related timing constraints for interconnected automotive systems at the design level: 1. To identify vulnerabilities of automotive systems under malicious attacks, we adopt and modify the behavioral model of a cooperative automotive system (CAS) [13] in UPPAALSMC by adding it with the models of an RSU-aided (RAISE) communication protocol in VANET and malicious attacks. The modification results in a refined behavioral model of the system, i.e., more details in terms of vehicular communication and security breaches are depicted; 2. Probabilistic extension of S/S timing constraints are specified in PrCCSL and the semantics of the extended constraints are translated into verifiable models with stochastic semantics for formal verification; 3. A set of mapping rules are proposed to facilitate the translation, based on which an automatic translation tool ProTL is implemented; 
4. Formal verification is performed on the $\mathrm{S} / \mathrm{S}$ timing constraints using UPPAALSMC under different attack scenarios.

The paper is organized as follows: Sect. 2 presents an overview of PrCCSL and UPPAAL-SMC. CAS is introduced as a running example in Sect. 3. Section 4.1 presents the UPPAAL-SMC model of CAS complemented with model of RAISE protocol and three types of attacks. S/S related timing constraints are specified in PrCCSL and translated into verifiable UPPAAL-SMC models in Sect. 5. The applicability of our approach is demonstrated by performing verification on CAS case study in Sect. 6 . Sections 7 and 8 present related works and conclusion.

\section{Preliminary}

In our framework, $\mathrm{S} / \mathrm{S}$ related timing constraints are specified in PrCCSL. UPPAAL-SMC is employed to perform formal verification on the timing constraints.

\subsection{Probabilistic Extension of Clock Constraint Specification Language (PrCCSL)}

PrCCSL [14] is a probabilistic extension of CCSL [3,23] for formal specification of timing constraints associated with stochastic behaviors. In PrCCSL, a clock represents a sequence of (possibly infinite) instants. An event is a clock and the occurrences of an event correspond to a set of ticks of the clock. PrCCSL provides two types of clock constraints, i.e., expressions and relations, to specify the progression/occurrences of clocks. An expression derives new clocks from the already defined clocks [3]. Let $c 1, c 2 \in C$, ITE (if-then-else) expression, denoted as $\beta$ ? $c 1: c 2$, defines a new clock that behaves either as $c 1$ or as $c 2$ according to the value of the boolean variable/formula $\beta$. DelayFor (denoted ref $(d) \rightsquigarrow$ base) results in a new clock by delaying the reference clock ref for $d$ ticks (or $d$ time units) of a base clock. FilterBy $(c \triangleq$ base $\boldsymbol{\nabla} u(v))$ builds a new clock $c$ by filtering the instants of a base clock according to a binary word $w=u(v)$, where $u$ is the prefix and $v$ is the period. " $(v)$ " denotes the infinite repetition of $v$. This expression results in a clock $c$ that $\forall k \in N^{+}$, if the $k^{t h}$ bit in $w$ is 1 , then at the $k^{\text {th }}$ tick of base, $c$ ticks.

A relation limits the occurrences among different events, which are defined based on run and history. A run corresponds to an execution of the system model where the clocks tick/progress. The history of a clock $c$ represents the number of times the clock $c$ has ticked prior to the current step.

Definition 1 (Run). A run $R$ consists of a finite set of consecutive steps where a set of clocks tick at each step $i$. The set of clocks ticking at step $i$ is denoted as $R(i)$, i.e., for all $i, 0 \leqslant i \leqslant n, R(i) \in R$, where $n$ is the number of steps of $R$.

Definition 2 (History). The history of clock $c$ in a run $R$ is a function: $H_{R}^{c}$ : $\mathbb{N} \rightarrow \mathbb{N}$. $H_{R}^{c}(i)$ indicates the number of times the clock $c$ has ticked prior to step $i$ in run $R$, which is initialized as 0 at step 0 . It is defined as: (1) $H_{R}^{c}(0)=0$; 
(2) $\forall i \in \mathbb{N}^{+}, c \notin R(i) \Longrightarrow H_{R}^{c}(i+1)=H_{R}^{c}(i)$; (3) $\forall i \in \mathbb{N}^{+}, c \in R(i) \Longrightarrow$ $H_{R}^{c}(i+1)=H_{R}^{c}(i)+1$.

A probabilistic relation in PrCCSL is satisfied if and only if the probability of the relation constraint being satisfied is greater than or equal to the probability threshold $p \in[0,1]$. Given $k$ runs $=\left\{R_{1}, \ldots, R_{k}\right\}$, the probabilistic subclock, coincidence, exclusion and precedence in PrCCSL are defined as follows:

Probabilistic Subclock: $c 1 \subseteq_{p} c 2 \Longleftrightarrow \operatorname{Pr}[c 1 \subseteq c 2] \geqslant p$, where $\operatorname{Pr}[c 1 \subseteq c 2]=$ $\frac{1}{k} \sum_{j=1}^{k}\left\{R_{j}=c 1 \subseteq c 2\right\}$, representing the ratio of runs that satisfies the relation out of $\mathrm{k}$ runs. A run $R_{j}$ satisfies the subclock relation between $c 1$ and $c 2$ "if $c 1$ ticks, $c 2$ must tick" holds at every step $i$ in $R_{j}$, s.t., $\left(R_{j} \models c 1 \subseteq c 2\right) \Longleftrightarrow(\forall i$ $0 \leqslant i \leqslant n, c 1 \in R(i) \Longrightarrow c 2 \in R(i))$. " $R_{j} \models c 1 \subseteq c 2$ " returns 1 if $R_{j}$ satisfies $c 1 \subseteq c 2$, otherwise it returns 0 .

Probabilistic Coincidence: $c 1 \equiv_{p} c 2 \Longleftrightarrow \operatorname{Pr}[c 1 \equiv c 2] \geqslant p$, where $\operatorname{Pr}[c 1 \equiv c 2]=$ $\frac{1}{k} \sum_{j=1}^{k}\left\{R_{j} \models c 1 \equiv c 2\right\}$, which represents the ratio of runs that satisfies the coincidence relation out of $\mathrm{k}$ runs. A run, $R_{j}$ satisfies the coincidence relation on $c 1$ and $c 2$ if the assertion holds: $\forall i, 0 \leqslant i \leqslant n,(c 1 \in R(i) \Longrightarrow c 2 \in R(i)) \wedge(c 2 \in$ $R(i) \Longrightarrow c 1 \in R(i))$. In other words, the satisfaction of coincidence relation is established when the two conditions "if $c 1$ ticks, $c 2$ must tick" and "if $c 2$ ticks, $c 1$ must tick" hold at every step.

Probabilistic Exclusion: $c 1 \#_{p} c 2 \Longleftrightarrow \operatorname{Pr}[c 1 \# c 2] \geqslant p$, where $\operatorname{Pr}[c 1 \# c 2]=$ $\frac{1}{k} \sum_{j=1}^{k}\left\{R_{j} \models c 1 \# c 2\right\}$, indicating the ratio of runs that satisfies the exclusion relation out of $\mathrm{k}$ runs. A run, $R_{j}$, satisfies the exclusion relation on $c 1$ and $c 2$ if $\forall i, 0 \leqslant i \leqslant n,(c 1 \in R(i) \Longrightarrow c 2 \notin R(i)) \wedge(c 2 \in R(i) \Longrightarrow c 1 \notin R(i))$, i.e., for every step, if $c 1$ ticks, $c 2$ must not tick and vice versa.

Probabilistic Precedence: $c 1 \prec p c 2 \Longleftrightarrow \operatorname{Pr}[c 1 \prec c 2] \geqslant p$, where $\operatorname{Pr}[c 1 \prec c 2]=$ $\frac{1}{k} \sum_{j=1}^{k}\left\{R_{j} \models c 1 \prec c 2\right\}$, which denotes the ratio of runs that satisfies the precedence relation out of k runs. A run $R_{j}$ satisfies the precedence relation if the condition $\forall i, 0 \leqslant i \leqslant n,\left(H_{R}^{c 1}(i) \geqslant H_{R}^{c 2}(i)\right)$ and $\left(H_{R}^{c 2}(i)=H_{R}^{c 1}(i)\right) \Longrightarrow(c 2 \notin R(i))$ hold, i.e., the history of $c 1$ is greater than or equal to the history of $c 2$, and $c 2$ must not tick when the history of the two clocks are equal.

\subsection{UPPAAL-SMC}

UPPAAL-SMC [31] performs the probabilistic analysis of properties by monitoring simulations of the complex hybrid system in a given stochastic environment and using results from the statistics to determine whether the system satisfies the property with some degree of confidence. UPPAAL-SMC provides a number of queries related to the stochastic interpretation of Timed Automata (STA) 
[8] and they are as follows, where $N$ and bound indicate the number of simulations to be performed and the time bound on the simulations respectively: 1. Probability Estimation estimates the probability of a requirement property $\phi$ being satisfied for a given STA model within the time bound: $\operatorname{Pr}[$ bound $] \phi$; 2. Hypothesis Testing checks if the probability of $\phi$ is satisfied within a certain probability $P_{0}: \operatorname{Pr}[$ bound $] \phi \geq P_{0}$; 3. Simulations: UpPaAL-SMC runs multiple simulations on the STA model and the $k$ (state-based) properties/expressions $\phi_{1}, \ldots, \phi_{k}$ are monitored and visualized along the simulations: simulate $N[\leq$ bound $]\left\{\phi_{1}, \ldots, \phi_{k}\right\}$.

\section{Running Example}

A cooperative automotive system (CAS) [13] is adopted to illustrate our approaches. CAS includes distributed and coordinated sensing, control, and actuation over three vehicles (denoted as $v_{i}$, where $i \in\{0,1,2\}$ ) which are running in the same lane. As shown in Fig. 1, a lead vehicle $\left(v_{0}\right)$ runs automatically by recognizing traffic signs on the road. The following vehicle must set its desired velocity identical to that of its immediate preceding vehicle. Vehicles should maintain sufficient braking distance to avoid rear-end collision while remaining close enough to guarantee communication quality. Vehicle movement relies on availability of environmental information, e.g., traffic signs, obstacles, etc. The position of $v_{i}$ is represented by Cartesian coordinate $\left(x_{i}, y_{i}\right)$, where $x_{i}$ and $y_{i}$ are distances measured from the vehicle to the two fixed perpendicular lines, i.e., $\mathrm{x}$-axis and $\mathrm{y}$-axis, respectively.

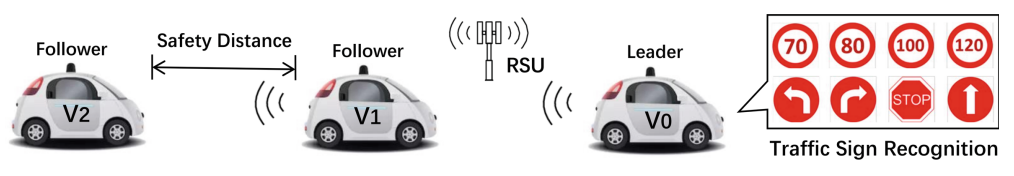

Fig. 1. Overview of Cooperative Automotive System

The cooperative driving of CAS requires prompt and secure information transmission among vehicles. We adopt a roadside unit aided (RAISE) [33] communication protocol in VANET to achieve the data transmission. Each vehicle periodically broadcasts its own position and velocity to its immediate following vehicle through wireless connection. The authentication of the identities of each vehicle and verification of messages sent by the vehicles is performed by RSU. For further details of RAISE, refer to Sect. 4.1. The following S/S properties on CAS are considered:

R1. The follower vehicle should not overtake its leading vehicle when the vehicles run at a positive direction of $\mathrm{x}$-axis.

R2. When the lead vehicle detects a stop sign, all the three vehicles must stop within a given time, e.g., $2000 \mathrm{~ms}$. 
R3. If the distance between a vehicle and its preceding vehicle is less than minimum safety distance, the vehicle should decelerate within a certain time $(200 \mathrm{~ms})$. $\mathrm{R} 4$. If the distance between a vehicle and its preceding vehicle is greater than the maximum safety distance (e.g., $100 \mathrm{~m}$ ), the vehicle should accelerate within a certain time, e.g., $300 \mathrm{~ms}$.

R5. When the lead vehicle starts to turn left (or turn right), the two follower vehicles should finish turning and run in the same lane within a given time.

R6. Authenticity: If a vehicle receives a message, its preceding vehicle must have sent a corresponding message before, i.e., the protocol should be resistant to message spoofing attack.

R7. Secrecy: Symmetric keys of vehicles should be kept confidential to attackers. R8. Integrity: The content of messages must not be modified during transmission, i.e., the protocol should be resistant to message falsification attack.

R9. Freshness: The vehicles should not accept an "obsolete" message, namely, the difference between the current time and the timestamp of the accepted message should be less than the predefined time threshold.

R10. The symmetric key agreement (i.e., mutual authentication) process between RSU and three vehicles should be completed within a certain time, e.g., $600 \mathrm{~ms}$. R11. A vehicle should send messages to its subsequent vehicle periodically with a period $200 \mathrm{~ms}$ and a jitter $100 \mathrm{~ms}$.

Among the above $\mathrm{S} / \mathrm{S}$ requirements, $\mathrm{R} 1-\mathrm{R} 5$ are safety [20] properties, which specify that the system should not cause undesirable results on its environment and aim at protecting human lives, health and assets from being damaged. R6R11 are security properties, which refer to the inability of the environment to affect the system in an undesirable way and aim to guarantee the confidentiality and integrity of transmitted information. The interdependencies among those S/S properties are conditional dependencies [17], i.e., violations of security properties can lead to the violations on safety properties. The events associated with those S/S properties can be interpreted as logical clocks in PrCCSL, which provides a way to express $\mathrm{S} / \mathrm{S}$ properties in the logical time manner [16]. Therefore, $\mathrm{S} / \mathrm{S}$ properties can be interpreted as logical timing constraints, i.e., the temporal and causality clock relations in PrCCSL.

The methodology for analysis of S/S related timing constraints in this paper can be generalized in Fig. 2. First, on the basis of the existing behavioral model of CAS described in [13], we enhance the CAS model by augmenting (parallelly composing) it with models of RAISE protocol and malicious attacks, resulting in a refined CAS model regarding vehicular communication characteristics and security-related adversary interference. Second, we specify S/S timing constraints (R1-R11) in PrCCSL and translate the PrCCSL specifications into corresponding STA and probabilistic queries. Finally, we combine the model of CAS and the STA of PrCCSL specifications, and perform formal verification based on the combined model using UPPAAL-SMC. 


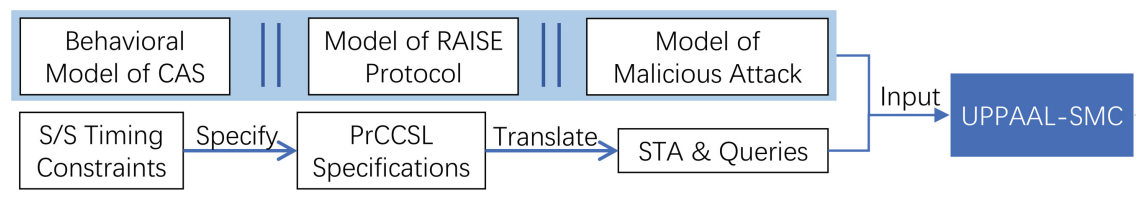

Fig. 2. Methodology for analysis of S/S timing constraints

\section{Modeling and Refinement of CAS in UPPAAL-SMC}

The behaviors of CAS are modeled as a network of stochastic timed automata (NSTA) in UPPAAL-SMC described in [13]. In this section, we refine the CAS model by adding it with the models of RAISE protocol and security attacks.

\subsection{Modeling of RAISE Protocol in UPPAAL-SMC}

We present a simplified version of RAISE protocol [33] and its UPPAAL-SMC model. The original RAISE protocol is modified to facilitate the communication mechanism of CAS, i.e., each follower vehicle receives messages from its immediate preceding vehicle and RSU. Furthermore, timing constraints are also appended to restrict the time duration of each step (e.g., encryption and decryption) during communication process. There are two phases in RAISE protocol, i.e., symmetric key agreement and information transmission.

1. Symmetric key agreement (SKA) is performed to obtain symmetric key $k_{i}$ for guaranteeing security of communication and generates pseudo identities $I D_{i}$ of vehicles for covering their real identities. The shared symmetric key between $R S U$ and $v_{i}$ is $k_{i}=g^{a b}$, where $g, a, b$ are three positive random numbers. As shown in Fig. 3, Encry $(m s g, k)(\operatorname{Decry}(m s g, k))$ denotes the encryption (decryption) of message $m s g$ with key $k$, where $k$ can be either a public key or symmetric key. $\operatorname{Sign}(m s g, k)$ generates signature of $m s g$ with a private key $k$. We use $P K_{i}$ to denote the public key of $v_{i}$ and $S K_{i}$ to represent the corresponding private key. "||" is the concatenation operation on messages.

Initially, $v_{i}$ randomly picks $g$ and $a$ (step 1 ), encrypts " $g \| a$ " and sends the encrypted result $\left(m_{i}\right)$ to $R S U$ (step 2). Upon receiving $m_{i}, R S U$ decrypts the message (step 3$)$. It then generates $b$ and $I D_{i}$, signs and sends the signed message $\left(r m_{i}\right)$ to $v_{i}$ (step 4 and 5). $v_{i}$ verifies the $r m_{i}$ 's signature (step 6) and sends back the signature of $g\|a\||| b \mid I D_{i}$ (step 7). Finally, $R S U$ verifies the signature $s_{i}$ (step $8)$. If all the steps are completed correctly, the key agreement process succeeds.

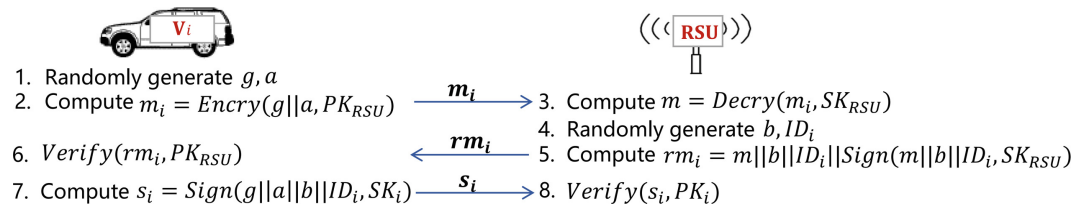

Fig. 3. Symmetric key agreement in RAISE 
2. Information transmission (IT) initiates after the SKA is completed. The traffic information (i.e, brake, direction, position and speed) of $v_{i}$ is integrated into a message msg $_{i}=$ brake $_{i} \|$ direction $_{i}\left\|x_{i}\right\| y_{i} \|$ speed $_{i}$. As presented in Fig. 4 , initially, $v_{i}$ generates the message authentication code (MAC) of $m s g_{i}$ with the symmetric key $k_{i}$ (generated in SKA). Then, $v_{i}$ concatenates the MAC code with

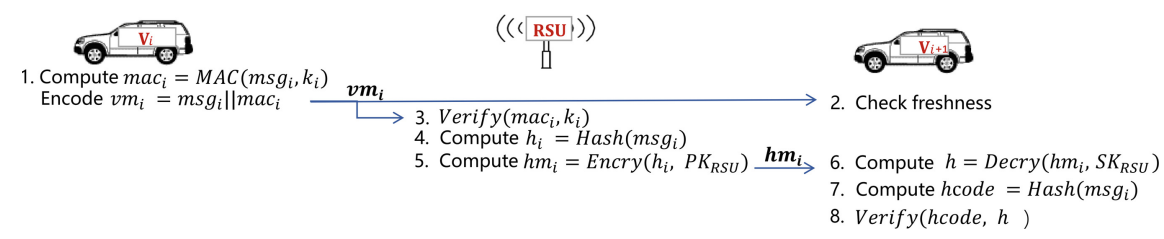

Fig. 4. Information transmission in RAISE

$m s g_{i}$ and sends it to $R S U$ and $v_{i+1}$ (step 1). Upon receiving $v m_{i}, v_{i+1}$ checks the freshness of the message (step 2), i.e., if the time interval between the current time and the time when $v m_{i}$ is sent is greater than the predefined threshold, $v_{i+1}$ drops $v m_{i}$. At the same time, $R S U$ checks the authenticity of $v m_{i}$ (step 3 ). If $m a c_{i}$ is correct, $R S U$ computes the hash code $h_{i}$ of message $m_{s} g_{i}$ (step 4). Afterwards, it encrypts $h_{i}$ and sends the encrypted result $h m_{i}$ to $v_{i+1}$ (step 5). $v_{i+1}$ decrypts $h m_{i}$ and get the hash code $h$ (step 6). Furthermore, to ensure the consistency of the message, $v_{i+1}$ itself also computes the hash code of $m s g_{i}$ (step 7). It then verifies whether the hash code calculated by itself is the same as the decrypted hash code and decides to accept or reject $m s g_{i}$ (step 8).

To model RAISE in UPPAAL-SMC, interactions among vehicles and RSU (i.e., sending/receiving messages) are modeled by synchronization channels [31] and global variables. The cryptographic operations in RAISE refer to public and private key encryption and decryption, i.e., a message encrypted by public key can be decrypted using the corresponding private key, and vice versa. The automaton of cryptographic device [6] is adopted to model the encryption and decryption. Figure 5 presents the STA capturing behaviors of vehicle $v_{i}$ and RSU in SKA. startEn (resp. startDe) and finDe (resp. finEn) are channels for indicating the starting and finishing of encryption (resp. decryption). The encryption/decryption result is denoted en_res/de_res. In the STA, names of locations indicate the corresponding steps pictured in Fig. 3.

IT phase from $v_{0}$ to $v_{1}$ is established with the help of RSU, modeled as the STA shown in Fig. 6 (the transmission from $v_{1}$ to $v_{2}$ can be modeled similarly). The behaviors of $v_{0}$ (sender), $v_{1}$ (receiver) and RSU in the IT phase are modeled in IT_v0, IT_v1 and IT_RSU STA, respectively.

The SKA (or IT) succeeds if each step of the SKA (IT) is completed correctly within a given time interval, modeled by invariant " $\mathrm{t} \leq \mathrm{d}$ " (the value of $d$ varies in different steps). If timeout occurs (i.e., " $\mathrm{t} \geq \mathrm{d}$ "), fail location will be activated and the procedure is restarted from the initial step. 

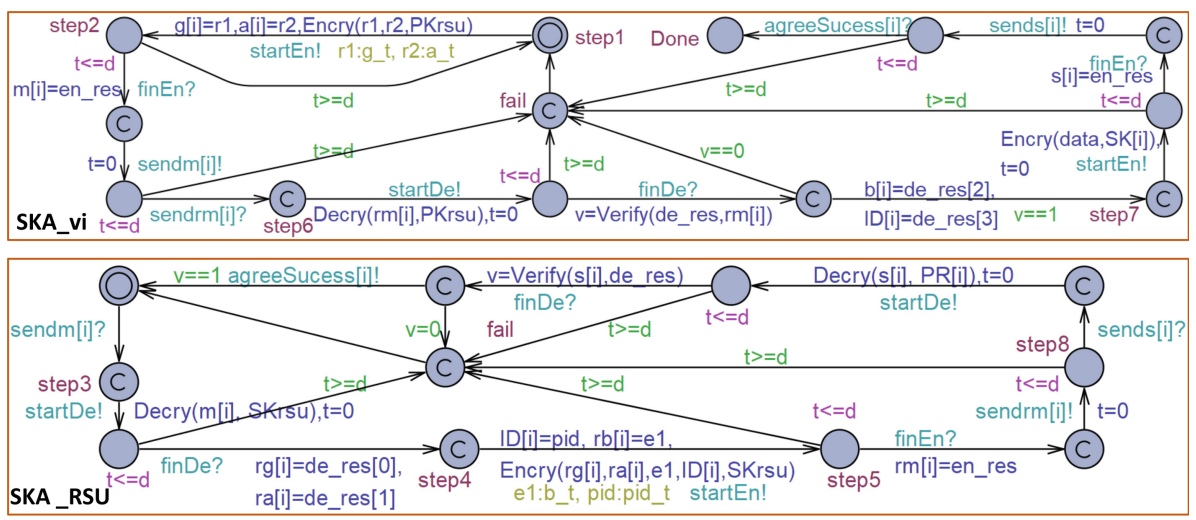

Fig. 5. UPPAAL-SMC model of SKA

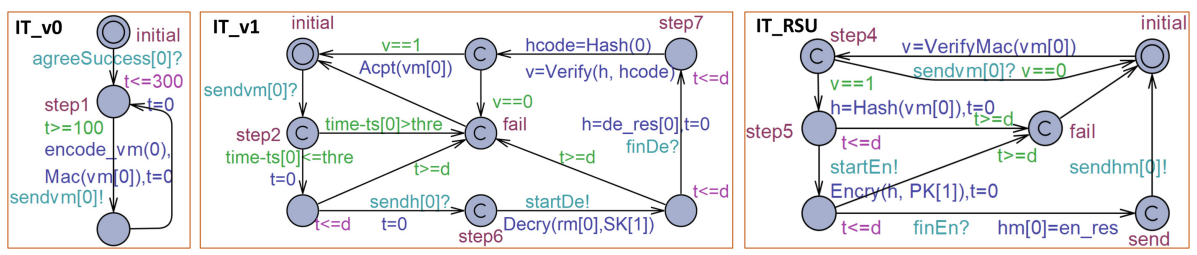

Fig. 6. UPPAAL-SMC model of IT

\subsection{Modeling of Attacks in UPPAAL-SMC}

We present the modeling of three types of attacks commonly used in the security analysis, i.e., message falsification, message replaying and message spoofing attacks [2]. The models of attacks are illustrated in Fig. 7, where the $l s$ parameter $(l s \in[0,100])$ serves as an indicator of level of adversarial strength while $q c$ $(q c \in[0,100])$ is an indicator of the adversarial channel quality.

Message Falsification Attack (MFA) aims to falsify messages transmitted from $v_{i}$ to $v_{i+1}$, which is modeled as MFA STA in Fig. 7. As described earlier, in RAISE, RSU verifies the authenticity of messages by checking the correctness of the MAC code of messages. To deceive the RSU on the validity of the modified message and avoid exposing itself to RSU, MFA attempts to obtain the symmetric key and utilizes the key to compute the MAC code of the falsified message. At s1 state, MFA eavesdrops on $r m_{i}$ (generated at step 5 in Fig. 3), which contains the information for symmetric key generation (i.e., $g, a, b)$. It tries to decrypt $r m_{i}$ when receiving it via sendrm $[i]$ ?. The probability that the decryption can succeed is $l s \%$, modeled by probabilistic choices [31] (dashed edges) with probability weight as $\frac{l s}{100}$ and $\frac{100-l s}{100}$. If the decryption succeeds, MFA obtains the symmetric key of $v_{i}$ based on the decrypted result (getKey(de_res)). Finally, it modifies the content of message using the key, and tries to send the modified message to $v_{i+1}(\operatorname{sendvm}[i]$ !). The probability that the message can be sent successfully is 
$(100-q c) \%$. In our setting, MFA modifies the speed $_{i}$ field in the message into a random value in $[100,120]$, and changes the direction as direction di $_{i}=4$, which indicates that the $v_{i}$ is running at the positive direction on y-axis.
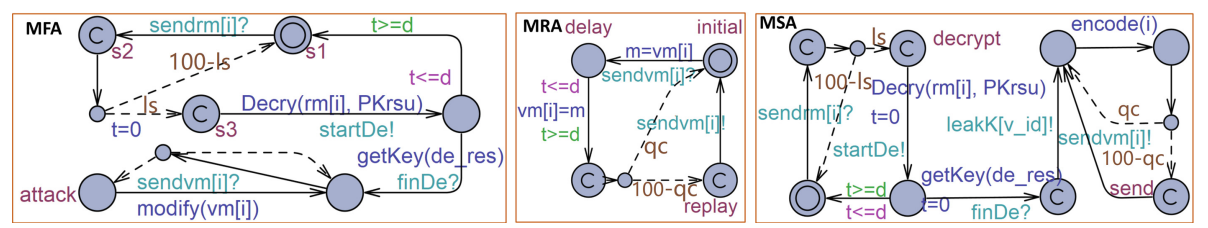

Fig. 7. STA of attacks

Message Replaying Attack (MRA) targets to replay obsolete messages that contain old information. The MRA STA represents an MRA that replays messages sent by $v_{i}$. Upon capturing a message (via sendvm[i]?), MRA stores the message $(m=v m[i])$ and tries to replay it at a later time (i.e., after $10 \mathrm{~s})$. The probability that the attacker can replay the message successfully is $(100-q c) \%$.

Message Spoofing Attack (MSA) impersonates a vehicle $\left(v_{i}\right)$ in order to inject fraudulent information into its subsequent vehicle $\left(v_{i+1}\right)$. Similar to MFA, MSA STA first obtains the symmetric key of $v_{i}$ by detecting and decrypting $r m_{i}$. It then fabricates a new message whose content is "brake $e_{i}=0$, speed $_{i}=$ 0 , direction $_{i}=4, x_{i}=0, y_{i}=10 "$ (denoted "encode(i)") and tries to send the message to $v_{i+1}(\operatorname{sendvm}[i]$ !), with the probability of the message being sent successfully as $(100-q c) \%$.

\section{Representation of S/S Related Timing Constraints in UPPAAL-SMC}

To enable the formal verification of $\mathrm{S} / \mathrm{S}$ related timing constraints (given in Sect. 3), we first investigate how to specify those constraints in PrCCSL. Then, translation from PrCCSL specifications of the constraints into verifiable STA is demonstrated. Furthermore, a tool ProTL that supports the automatic transformation based on the proposed translation rules is introduced.

\subsection{Specifications of S/S Related Timing Constraints in PrCCSL}

The specifications of R1-R11 are presented in Table 1, where $a c$ is a clock that always ticks while $n c$ represents a clock that never ticks. R1 is specified as an exclusion relation between $x$ dir (the event that the vehicles are running at the positive direction of $\mathrm{x}$-axis) and ovtake (the event that the position of follower $v_{1}$ on $\mathrm{x}$-axis is greater than that of leader $v_{0}$ ). Similarly, R7 and R9 can be specified as exclusion relations.

In the specification of $\mathrm{R} 2$, stopD is a clock generated by delaying stopSign (the event that the leader vehicle detects a stop sign) for $2000 \mathrm{~ms}$. vstop refers 
Table 1. PrCCSL specifications of R1-R11

\begin{tabular}{|c|c|}
\hline Req & PrCCSL Specification \\
\hline $\mathrm{R} 1$ & $x d i r \triangleq \operatorname{dir}=1 ?$ ac $:$ nc, ovtake $\triangleq x_{1} \geq x_{0} ?$ ac $: n c, x d i r \#_{0.95}$ ovtake \\
\hline $\mathrm{R} 2$ & $\begin{array}{l}\text { stopSign } \triangleq \text { sign }=5 ? \text { signRec }: \text { nc, stopD } \triangleq \text { stopSign }(2000) \rightsquigarrow m s, \\
\text { vstop } \preceq_{0.95} \text { stopD }\end{array}$ \\
\hline R3 & $v$ UnsafeDe $\triangleq v$ Unsafe $(200) \rightsquigarrow m s, v D e c \prec_{0.95}$ vUnsafeDe \\
\hline $\mathrm{R} 4$ & $v$ FarDisDe $\triangleq v$ FarDis $(300) \rightsquigarrow m s$, startAcc $\prec_{0.95}$ vFarDisDe \\
\hline R5 & v0TurnDe $\triangleq v 0 T u r n(3000) \rightsquigarrow m s$, finTurn $\preceq_{0.95}$ v0TurnDe \\
\hline $\mathrm{R} 6$ & msgRec $\subseteq_{0.95}$ msgSent \\
\hline $\mathrm{R} 7$ & leakK $\#_{0.95} a c$ \\
\hline $\mathrm{R} 8$ & validMsg $\triangleq r M s g=s M s g ? m s g R e c: n c, m s g R e c \equiv_{0.95}$ validMsg \\
\hline $\mathrm{R} 9$ & oldMsg $\triangleq$ time $-t s>$ thre $?$ msgAcpt $:$ nc, msgAcpt $\#_{0.95}$ oldMsg \\
\hline R10 & start $S K A D e \triangleq \operatorname{start} S K A(600) \rightsquigarrow m s$, fin $S K A \prec_{0.95}$ start $S K A D e$ \\
\hline R11 & $\begin{array}{l}f c l k \triangleq m s g \text { Sent } \nabla 01(1), \text { sentDe } 1 \triangleq m s g \text { Sent }(100) \rightsquigarrow m s \\
\text { sentDe } 2 \triangleq m s g \text { Sent }(300) \rightsquigarrow m s, \text { sentDe } 1 \preceq_{0.95} \mathrm{fclk} \\
\mathrm{fclk} \preceq_{0.95} \text { sentDe } 2\end{array}$ \\
\hline
\end{tabular}

to the event that three vehicles are completely stopped, which should occur no later than stopD. Hence, $\mathrm{R} 2$ is expressed as a causality relation between vstop and stopD. R3-R5 can be specified in a similar manner.

R6 (authenticity) is expressed as a subclock relation between msgRec and msgSent, where msgRec (msgSent) represents the event that a message is received (sent) by the follower (leader) vehicle. $\mathrm{R} 8$ is specified as a coincidence relation between msgRec and validMsg, where validMsg is a clock that ticks with $m s g R e c$ when the received message $r M s g$ is identical with the sent message $s M s g$ (i.e., $r M s g==s M s g$ ). For R10, startSKA ( $\operatorname{finSKA}$ ) represents the starting (completion) of SKA. startSKADe is a clock constructed by delaying startSKA for $600 \mathrm{~ms}$. R10 delimits that finSKA must occur before startSKADe. R11 states that two consecutive occurrences of msgSent must has a interval of [period jitter, period + jitter $] \mathrm{ms}$ (i.e., $[100,300] \mathrm{ms}$ ). In the specification of R11, fclk is a clock generated by filtering out the $1^{\text {st }}$ tick of msgSent. sentDe 1 and sentDe2 are two clocks generated by delaying msgSent for $100 \mathrm{~ms}$ and $300 \mathrm{~ms}$. R11 can be interpreted as: $\forall i \in \mathbb{N}^{+}$, the $i^{\text {th }}$ tick of $f c l k$ should occur later than the $i^{\text {th }}$ tick of sentDe1 but prior to the $i^{t h}$ tick of sentDe2.

\subsection{Translation of PrCCSL into STA}

We present how the S/S related timing constraints specified in PrCCSL can be transformed into STA and probabilistic queries in UPPAAL-SMC. We first describe how clock tick and history (introduced in Sect. 2) can be represented in UPPAAL-SMC. Using the mapping, we then demonstrate that expressions and relations in PrCCSL can be translated into STA and queries. 
In the earlier work [14], the semantics of PrCCSL operators are translated into STA based on discrete time, i.e., the continuous physical time is discretized into a set of equalized steps. As a result, two clock instants are still considered coincident even if they are one time step apart. To alleviate this restriction and enable the representation of PrCCSL that pertains to continuous real-time semantics, the mapping patterns are refined: two clock instants are coinstantaneous only if the time difference between them is insignificant, i.e., the time difference between them is less than a positive infinitesimal value $e$, e.g., $e=0.000001$.

In PrCCSL, a logical clock represents an event and the instants of the clock correspond to the occurrences of the event. A logical clock $c$ is represented as a synchronization channel $c$ ! in UPPAAL-SMC. The history of $c$ is modeled as the STA shown in Fig. 8: whenever $c$ occurs $(c$ ?), the value of its history is increased by 1 (i.e., $h++$ ).

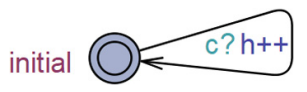

Fig. 8. History

Based on the mapping patterns of tick and history, the PrCCSL expressions (including ITE, DelayFor and filterBy), as well as relations (including subclock, coincidence, exclusion and precedence), can be represented as STA and queries shown in Fig. 9.

The STA of expressions trigger the ticks of the new clock (denoted res!) based on the occurrences of existed clocks. To represent relations, observer STA that capture the semantics of standard subclock, coincidence, exclusion and precedence relations are constructed. Each observer STA contains a "fail" location (see Fig. 9), which indicates the violation of the corresponding relation. Recall the definition of PrCCSL in Sect.2, the probability of a relation being satisfied is interpreted as a ratio of runs that satisfies the relation among all runs. It is specified as Hypothesis Testing queries in UPPAAL-SMC, $H_{0}: \frac{m}{k} \geqslant p$ against $H_{1}: \frac{m}{k}<p$, where $m$ is the number of runs satisfying the given relation out of all $k$ runs. As a result, the probabilistic relations are interpreted as the query (see Fig. 9): $\operatorname{Pr}[$ bound $]([] \neg S T A$.fail) $\geq p$, which means that the probability of the "fail" location of the observer STA never being reached should be greater than or equal to $p$. The STA of expressions and relations are composed to the system NSTA in parallel. Then, the probabilistic analysis is performed over the composite NSTA that enables us to verify the S/S related timing constraints over the entire system using UPPAAL-SMC.

Tool support: Manual translation of PrCCSL specifications into UPPAAL models for verification can be time-consuming and error-prone. To improve the accuracy and efficiency of translation, we implement a tool ProTL (ProbabilisticCCSL TransLator) [26] that provides a push-button transformation from PrCCSL specifications into corresponding STA \& queries. Furthermore, verification and simulation support is provided in ProTL by employing the UPPAAL-SMC as the backend analysis engine. ProTL encompasses the following features: (1) An editor for editing PrCCSL specification of requirements (stored as ".txt" files); (2) Automated transformation of PrCCSL specifications into UPPAAL-SMC STA; (3) Integration of the STA and the system behavioral model (imported by users); (4) A configuration palette for setting parameters (e.g., time bound of simula- 


\begin{tabular}{|c|c|}
\hline & Remarks \\
\hline 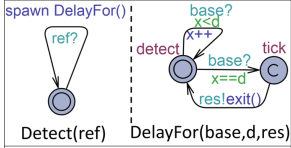 & $\begin{array}{l}\text { DelayFor: res } \triangleq r e f(d) \rightsquigarrow \text { base } \\
\text { When ref occurs (ref?), its DelayFor STA is spawned by Detect STA. The spawned STA stays } \\
\text { in the detect location until base ticks } d \text { times. When base ticks } d \text { times }(x==\mathrm{d}) \text {, it transits to } \\
\text { the tick location and triggers res (res!). Then it becomes inactive (denoted "exit(l)"), i.e., } \\
\text { calculation of the current tick of res is completed. }\end{array}$ \\
\hline & $\begin{array}{l}\text { ITE (if-then-else): res } \triangleq b ? c 1: c 2 \\
\text { ITE generates a new clock res that behaves either as } c 1 \text { or as } c 2 \text { base on the value of } \\
\text { boolean variable } b \text {. If } b \text { is true }(b==1) \text {, the tick of res will be triggered (denoted res!) } \\
\text { whenever } c 1 \text { occurs (c1?). Otherwise, res ticks with } c 2 \text { when } b \text { is false }(b==0) \text {. }\end{array}$ \\
\hline prefix & $\begin{array}{l}\text { FilterBy: res } \triangleq \text { base } \nabla u(v) \\
\text { FilterBy filters the instants of base based on a binary word } w=u(v) \text {, i.e., } \forall \mathrm{k} \in \mathrm{N} \text {, if the } k t h \\
\text { bit in } w \text { is } 1 \text {, then at the } k \text { th tick of base, res ticks. } u \text { and } v \text { are two boolean arrays. I } u \text { and } l v \\
\text { represent the size of } u \text { and } v \text {. As base ticks (base?), the STA firstly traverses the bits in } u \text { (at } \\
\text { prefix state) and then iterates the bits in } v \text { (at period state). If the present bit (indicated by } \\
\text { the index) of the binary word is } 1 \text {, the STA triggers res (res!). Otherwise, it moves to the } \\
\text { initial state, updates the index to refer to the next bit of } w(i++/ \mathrm{j}++) \text { and repeats the process. }\end{array}$ \\
\hline & $\begin{array}{l}\text { Probabilistic Coincidence: } c 1 \equiv{ }_{p} c 2 \\
\text { When } c 1(c 2) \text { ticks via } c 1 \text { ? }(c 2 \text { ?), the STA checks if the other clock, } c 2 \text { ( } c 1) \text {, ticks at the same } \\
\text { time. If } c 2 \text { (c1) occurs within a positive infinitesimal value ( }<<=e) \text {, the STA transits to success } \\
\text { location. Otherwise, the coincidence relation is violated and STA transits to fail location. } \\
\text { Probabilistic coincidence is expressed as: } \operatorname{Pr}[\text { bound]([ ] - Coincidence.fail) } \geq p \text {. }\end{array}$ \\
\hline & $\begin{array}{l}\text { Probabilistic Subclock: } c 1 \subseteq_{p} c 2 \\
\text { The relation limits that } c 2 \text { (superclock) must tick when } c 1 \text { (subclock) ticks, i.e., when } c 1 \text { ticks, } \\
\text { c2 must coincide with } c 1 \text {. When } c 1 \text { (c2) occurs, the STA checks whether the other clock also } \\
\text { ticks at the same time. When } c 1 \text { (subclock) ticks but } c 2 \text { does not occur (within e time unit), } \\
\text { the relation is violated and the STA transits to fail location. Probabilistic subclock is } \\
\text { expressed as: } \operatorname{Pr}[\text { bound] }[[]-\text { Subclock.fail) } \geq p \text {. }\end{array}$ \\
\hline & $\begin{array}{l}\text { Probabilistic Exclusion: } c 1 \#_{p} c 2 \\
\text { When } c 1(c 2) \text { ticks via } c 1 \text { ? ( }(c 2 \text { ?), the STA checks if the other clock, } c 2 \text { ( } c 1) \text {, ticks at the same } \\
\text { tme, i.e., whether } c 1(c 2) \text { occurs or not when } t \leq e \text {. If it occurs, the exclusion relation is } \\
\text { violated and STA moves to fail location. Probabilistic exclusion is expressed as: Pr[bound][[] } \\
- \text { Exclusion.fail) } \geq p \text {. }\end{array}$ \\
\hline Precedence (c2, h1, & $\begin{array}{l}\text { Probabilistic Precedence: } c 1 \prec_{p} c 2 \\
\text { The relation states that } c 1 \text { must run faster than } c 2 \text {, i.e., the history of } c 1 \text { (h1) must be } \\
\text { greater than or equal to the history of } c 2(h 2) \text {, and } c 2 \text { must not tick when the histories of } \\
\text { the two clocks are equal. Therefore, if } c 1 \text { ticks via } c 1 \text { ? and } c 1 \text { runs slower (i.e., h1<h2), or } \\
c 2 \text { ticks via } c 2 \text { ? when their histories are equal (h1=h2), the precedence relation is violated } \\
\text { and fail location is activated. Probabilistic precedence is expressed as: Pr[bound]([ ] - } \\
\text { Precedence.fail) } \geq p \text {. }\end{array}$ \\
\hline
\end{tabular}

Fig. 9. STA of PrCCsL operators

tion, number of simulations) used for verification and simulation; (5) Automatic generation of probabilistic queries (introduced in Sect. 2) based on user-specified parameters; (6) Capability of performing verification and simulation on PrCCSL specifications against the integrated model and generated queries.

The GUI of ProTL is implemented by applying the Python package TKINTER [27]. The implementation of Translator is achieved by the ANother Tool for Language Recognition (ANTLR) [24], a parser generator that can constructs lexical parsers for a language by analyzing user-defined syntax of the language. We specified the syntax of PrCCSL in Backus-Naur Form (BNF) and apply ANTLR to generate a parser that can analyze and recognize encodings in the format of PrCCSL. The parser reads the PrCCSL specifications and generates abstract syntax trees (AST), i.e., an intermediate form that has tree structures. 
By traversing AST, the information (i.e., operators and parameters) of PrCCSL can be extracted and utilized for generation of corresponding STA.

\section{Experiment}

To identify vulnerabilities of system to external malicious attackers, we combine the refined CAS system model (including the models of RAISE protocol) with models of three different attackers. Formal verification on S/S related timing constraints (R1-R11) for the combined model is performed by UPPAAL-SMC. The combined CAS model contains the stochastic behaviors in terms of the unpredictable environments (e.g., the traffic signs are randomly recognized by the leader vehicle of CAS and the probability of each sign type occurring is equally set as $16.7 \%$ ), as well as the indeterministic behaviors modeled by weighted probability choices in the STA of attacks (see Fig. 7). In our setting, $l s$ and $q c$ are configured as 10 and 90, respectively. To estimate the probability of an attack being launched on CAS successfully, Probability Estimation query is applied to check the probability that the "attack" location in each attack STA is reachable from the system NSTA. The time bound of the verification is set as 10000 . The probability of message falsification, message replaying and message spoofing attack being successfully completed by the corresponding attacker is within the range of $[0.109,0.209],[0.563,0.663]$ and $[0.143,0.243]$, respectively.

In our experiments, $\mathrm{S} / \mathrm{S}$ related timing constraints are specified in PrCCSL and transformed into STA using ProTL. Each constraint is specified as a PrCCSL relation (as described in Sect. 5.1) whose probability threshold is $95 \%$. The verification results are demonstrated in Table 2, in which " $\sqrt{ }$ " denotes the corresponding requirement is satisfied while " $x$ " indicates the violation of the requirement: Under the message replaying attack, all the S/S timing constraints are established as valid with $95 \%$ level of confidence. In the message falsification attack, the secrecy and integrity properties (R7 and R8), as well as three safety properties (R3-R5), are violated. The MSA damages the authenticity (R6) and secrecy (R7) of communication, and leads to the violations of four safety properties, i.e., $\mathrm{R} 1$ and R3-R5.

Table 2. Verification results of timing constraints under different attacks

\begin{tabular}{|c|c|c|c|c|c|c|c|c|c|c|c|c|c|c|}
\hline Attacks & R1 & R2 & R3 & R4 & R5 & R6 & R7 & R8 & R9 & R10 & R11 & Average Time & Mem $(\mathrm{Mb})$ \\
\hline Message Falsification & $\sqrt{ }$ & $\sqrt{ }$ & $\times$ & $\times$ & $\times$ & $\sqrt{ }$ & $\times$ & $\times$ & $\sqrt{ }$ & $\sqrt{ }$ & $\sqrt{ }$ & 40.20 & 57.94 \\
\hline Message Replaying & $\sqrt{ }$ & $\sqrt{ }$ & $\sqrt{ }$ & $\sqrt{ }$ & $\sqrt{ }$ & $\sqrt{ }$ & $\sqrt{ }$ & $\sqrt{ }$ & $\sqrt{ }$ & $\sqrt{ }$ & $\sqrt{ }$ & 68.33 & 61.49 \\
\hline Message Spoofing & $\times$ & $\sqrt{ }$ & $\times$ & $\times$ & $\times$ & $\times$ & $\times$ & $\sqrt{ }$ & $\sqrt{ }$ & $\sqrt{ }$ & $\sqrt{ }$ & 58.11 & 40.23 \\
\hline
\end{tabular}

The experiment results indicate the severity of impacts on safety and security caused by the demonstrated attacks on CAS: No requirement is violated under MRA scenario while the MSA causes the violations of most safety properties. 
When CAS is attached with the STA of MSA or MFA, the secrecy of symmetric key is violated. With the obtained symmetric key, MSA can masquerade message as legitimate vehicles and MFA is able to tamper the content of messages without being detected, leading to the violations of authenticity (R6) and integrity (R7) respectively. To explore how the malicious attackers can influence the safety of system, we conduct simulation by using Simulations queries. The simulation results in Fig. 10 illustrate how an MSA drives the system to undesirable states.

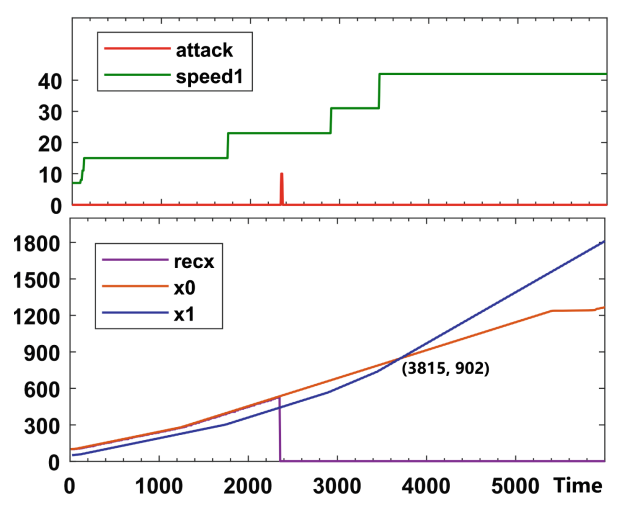

(a) R1

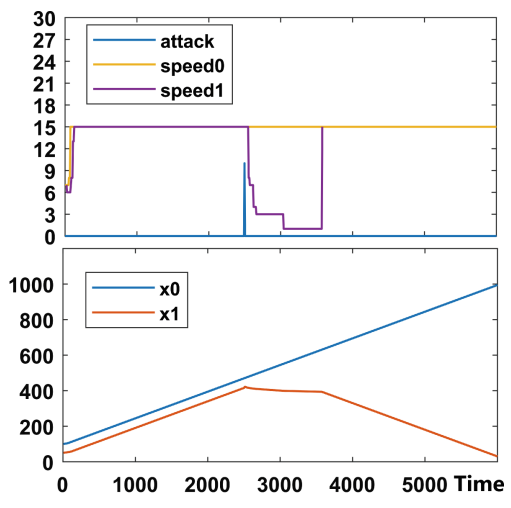

(b) R4

Fig. 10. Simulation results of R1 and R4: (a) At Time $=2345$, the attack occurs (indicated by the rising edge of the red line). MSA sends the fabricated position information of $V_{0}$ to $V_{1}$ (the value of recx becomes 0 ), which tricks $V_{1}$ to think that the distance between $V_{0}$ and $V_{1}$ exceeds the maximum limit. $V_{1}$ keeps increasing its speed (speed1) and thus leading to the collision (indicated by $x_{0}==x_{1}$ ) at Time $=3815$, which violates R1. (b) When an attack takes place at Time $=2496$ (indicated by the rising edge of the blue line), $V_{1}$ receives the message from the attacker and is deluded into believing that the speed of $V_{0}$ is 0 . Therefore, $V_{1}$ keeps decreasing its speed even if the distance between $V_{0}$ and $V_{1}$ becomes greater than $100 \mathrm{~m}$, which violates $\mathrm{R} 4$. (Color figure online)

\section{Related Work}

Formal verification of (non)-functional properties of automotive systems containing stochastic behaviors were investigated in several works [13-15]. In these works, systems are by default resilient to security threats and the safety properties are analyzed under no malicious attack scenarios, which is inadequate for design of automotive systems interconnected via wireless communications. Combined analysis of safety and security $(\mathrm{S} / \mathrm{S})$ properties for interconnected cyber physical systems have been addressed in earlier works $[1,21,29]$, which are however, limited to theoretical frameworks and high-level descriptions of S/S properties without the support for formal verification. Pedroza et al. [25] proposed a SysML based environment called AVATAR for the formal verification of S/S properties, which enables assessment of the impacts of cyber-security threats on 
functional safety. Wardell et al. [32] proposed an approach for identifying security vulnerabilities of industrial control systems by modeling malicious attacks as PROMELA models amenable to formal verification. However, those approaches lack precise probabilistic annotations specifying stochastic properties regarding to S/S aspects. Kumar et al. [18] introduced the attack-fault trees formalism for descriptions of attack scenarios and conducted formal analysis by using UPPAALSMC to obtain quantitative estimation on impacts of system failures or security threats. On the other hand, our work is based on the probabilistic extension of $\mathrm{S} / \mathrm{S}$ related timing constraints with the focus on probabilistic verification of the extended constraints.

\section{Conclusion}

This paper presents a model-based approach for probabilistic formal analysis of safety and security (S/S) related timing constraints for interconnected automotive system in EAST-ADL at the early design phase. The behavioral model of automotive system in UPPAAL-SMC is refined by adding the models of vehicular communication protocol and malicious attacks, which facilitates to exploit the impacts of adversary environment on S/S of the system. Timing constraints are specified in PrCCSL and translated into stochastic timed automata (STA) amenable to formal verification using UPPAAL-SMC. A set of translation rules from PrCCSL to STA, as well as the corresponding tool support for automating the translation are provided. We demonstrate our approach by performing formal verification on a cooperative automotive system (CAS) case study. Although, we have shown the one-to-one mapping patterns from a subset of PrCCSL operators to STA for conducting formal verification on timing constraints using UPPAALSMC, as ongoing work, systematic and formal translation techniques covering a full set of PrCCSL constraints are further studied. Furthermore, new features of ProTL with respect to analysis of UPPAAL-SMC models involving wider range of variable/query types (e.g., urgent channels, bounded integers) are further developed.

Acknowledgment. This work is supported by the EASY project funded by NSFC, a collaborative research between Sun Yat-Sen University and University of Southern Denmark.

\section{References}

1. Abdo, H., Kaouk, M., Flaus, J.M., Masse, F.: A safety/security risk analysis approach of industrial control systems: a cyber bowtie-combining new version of attack tree with bowtie analysis. Comput. Secur. 72, 175-195 (2018)

2. Amoozadeh, M., et al.: Security vulnerabilities of connected vehicle streams and their impact on cooperative driving. IEEE Commun. Mag. 53(6), 126-132 (2015)

3. André, C.: Syntax and semantics of the clock constraint specification language (CCSL). Ph.D. thesis, Inria (2009) 
4. Bernat, G., Burns, A., Llamosi, A.: Weakly hard real-time systems. Trans. Comput. 50(4), 308-321 (2001)

5. Blom, H., et al.: TIMMO-2-USE timing model, tools, algorithms, languages, methodology, use cases. Technical report, TIMMO-2-USE (2012)

6. Corin, R., Etalle, S., Hartel, P.H., Mader, A.: Timed model checking of security protocols. In: ACM Workshop on Formal Methods in Security Engineering (FMSE), pp. 23-32. ACM (2004)

7. David, A., et al.: Statistical model checking for stochastic hybrid systems. In: Hybrid Systems and Biology (HSB), pp. 122-136. EPTCS (2012)

8. David, A., Larsen, K.G., Legay, A., Mikučionis, M., Poulsen, D.B.: UPPAAL-SMC tutorial. Int. J. Softw. Tools Technol. Transf. 17(4), 397-415 (2015)

9. EAST-ADL: EAST-ADL specification v2.1.9. Technical report, MAENAD (2011). https://www.maenad.eu/public/EAST-ADL-Specification_M2.1.9.1.pdf

10. Engoulou, R.G., Bellaïche, M., Pierre, S., Quintero, A.: VANET security surveys. Comput. Commun. 44, 1-13 (2014)

11. IEC 61508: Functional safety of electrical electronic programmable electronic safety related systems (2010)

12. ISO 26262-6: Road vehicles functional safety part 6 . Product development at the software level (2011)

13. Kang, E.Y., Huang, L., Mu, D.: Formal verification of energy and timed requirements for a cooperative automotive system. In: ACM/SIGAPP Symposium On Applied Computing (SAC), pp. 1492-1499. ACM (2018)

14. Kang, E.-Y., Mu, D., Huang, L.: Probabilistic verification of timing constraints in automotive systems using UPPAAL-SMC. In: Furia, C.A., Winter, K. (eds.) IFM 2018. LNCS, vol. 11023, pp. 236-254. Springer, Cham (2018). https://doi.org/10. 1007/978-3-319-98938-9_14

15. Kang, E.Y., Mu, D., Huang, L., Lan, Q.: Verification and validation of a cyberphysical system in the automotive domain. In: IEEE International Conference on Software Quality, Reliability and Security Companion (QRS), pp. 326-333. IEEE (2017)

16. Khan, A.M., Mallet, F., Rashid, M.: Combining SysML and MARTE/CCSL to model complex electronic systems. In: Information Systems Engineering (ICISE), pp. 12-17. IEEE (2016)

17. Kriaa, S., Pietre-Cambacedes, L., Bouissou, M., Halgand, Y.: A survey of approaches combining safety and security for industrial control systems. Reliab. Eng. Syst. Saf. 139, 156-178 (2015)

18. Kumar, R., Stoelinga, M.: Quantitative security and safety analysis with attackfault trees. In: High Assurance Systems Engineering (HASE), pp. 25-32. IEEE (2017)

19. Legay, A., Viswanathan, M.: Statistical model checking: challenges and perspectives. Int. J. Softw. Tools Technol. Transf. 17(4), 369-376 (2015)

20. Line, M.B., Nordland, O., Røstad, L., Tøndel, I.A.: Safety vs. Security. In: International Conference on Probabilistic Safety Assessment and Management (PSAM) (2006)

21. Macher, G., Höller, A., Sporer, H., Armengaud, E., Kreiner, C.: A combined safetyhazards and security-threat analysis method for automotive systems. In: Koornneef, F., van Gulijk, C. (eds.) SAFECOMP 2015. LNCS, vol. 9338, pp. 237-250. Springer, Cham (2015). https://doi.org/10.1007/978-3-319-24249-1_21

22. MAENAD (2011). http://www.maenad.eu/

23. Mallet, F., De Simone, R.: Correctness issues on MARTE/CCSL constraints. Sci. Comput. Program. 106, 78-92 (2015) 
24. Parr, T.: The definitive ANTLR 4 reference. Pragmatic Bookshelf (2013)

25. Pedroza, G., Apvrille, L., Knorreck, D.: Avatar: a SysML environment for the formal verification of safety and security properties. In: New Technologies of Distributed Systems (NOTERE), pp. 1-10. IEEE (2011)

26. ProTL. https://sites.google.com/view/protl

27. Tkinter: Python interface to Tcl/Tk. https://docs.python.org/3/library/tkinter. html

28. Raya, M., Hubaux, J.P.: Securing vehicular Ad Hoc networks. J. Comput. Secur. 15(1), 39-68 (2007)

29. Sabaliauskaite, G., Mathur, A.P.: Aligning cyber-physical system safety and security. In: Cardin, M.A., Krob, D., Lui, P., Tan, Y., Wood, K. (eds.) Complex Systems Design \& Management Asia, pp. 41-53. Springer, Cham (2015). https://doi.org/ 10.1007/978-3-319-12544-2_4

30. Specification, O.: UML profile for MARTE: modeling and analysis of real-time embedded systems. Technical report, Object Management Group (2011)

31. UPPAAL-SMC. http://people.cs.aau.dk/ adavid/smc/

32. Wardell, D.C., Mills, R.F., Peterson, G.L., Oxley, M.E.: A method for revealing and addressing security vulnerabilities in cyber-physical systems by modeling malicious agent interactions with formal verification. Proc. Comput. Sci. 95, 24-31 (2016)

33. Zhang, C., Lin, X., Lu, R., Ho, P.H., Shen, X.: An efficient message authentication scheme for vehicular communications. IEEE Trans. Veh. Technol. 57(6), 3357-3368 (2008)

Open Access This chapter is licensed under the terms of the Creative Commons Attribution 4.0 International License (http://creativecommons.org/licenses/by/4.0/), which permits use, sharing, adaptation, distribution and reproduction in any medium or format, as long as you give appropriate credit to the original author(s) and the source, provide a link to the Creative Commons license and indicate if changes were made.

The images or other third party material in this chapter are included in the chapter's Creative Commons license, unless indicated otherwise in a credit line to the material. If material is not included in the chapter's Creative Commons license and your intended use is not permitted by statutory regulation or exceeds the permitted use, you will need to obtain permission directly from the copyright holder. 